\title{
Narkolepsie im Erwachsenenalter: Definition, Ätiologie und Behandlung
}

\section{Narcolepsy in adults: Definition, etiology and treatment}

\author{
Autoren \\ Michael Malter ${ }^{1}$, Janina Neuneier ${ }^{1}$, Annika Triller², Ulf Kallweit ${ }^{2}$ \\ Institute \\ 1 Universität Köln, Klinik und Poliklinik für Neurologie \\ 2 Zentrum für Narkolepsie/Hypersomnien, Klin. Schlaf- und \\ Neuroimmunologie, Institut für Immunologie, Universität \\ Witten/Herdecke
}

Schlüsselwörter

Narkolepsie, Hypocretin, Solriamfetol, Pitolisant, Methylphenidat, Natrium-Oxybat

Keywords

Narcolepsy, Hypocretin, Solriamfetol, Pitolisant, Methylphenidate, Sodium oxybate

eingereicht 26.06 .2020

akzeptiert $\quad 17.08 .2020$

Bibliografie

Fortschr Neurol Psychiatr 2021; 89: 103-113

DOI 10.1055/a-1244-2612

ISSN 0720-4299

(C) 2020. Thieme. All rights reserved.

Georg Thieme Verlag KG, Rüdigerstraße 14,

70469 Stuttgart, Germany

Korrespondenzadresse

PD Dr. med. Michael Malter

Klinik und Poliklinik für Neurologie, Uniklinik Köln

Kerpener Straße 61

50937 Köln

Deutschland

Tel.: 0221-478-4015

Fax: 0221/478-5669

E-Mail: michael.malter@uk-koeln.de

\section{ZUSAMMENFASSUNG}

Narkolepsie ist eine Hypersomnolenz-Erkrankung, die mit einer Störung der Wach-Schlaf-Regulation einhergeht.
Leitsymptome sind exzessive Tagesschläfrigkeit und Kataplexien. Zudem treten hypnagogene/hypnopompe Halluzinationen, Schlaflähmungen und gestörter Nachtschlaf auf. Es werden zwei Formen unterschieden. Bei der Narkolepsie Typ 1, früher Narkolepsie mit Kataplexie, besteht eine Hypocretin-Defizienz. Die Ursache der Narkolepsie Typ 2, früher Narkolepsie ohne Kataplexie, ist im Wesentlichen ungeklärt. Zur Diagnosestellung ist ein multimodaler Ansatz notwendig. Die Latenz zwischen Erkrankungsbeginn und Diagnosestellung in Europa beträgt im Mittel 14 Jahre. Es bestehen erhebliche Probleme in der Versorgung von Menschen mit Narkolepsie. Die in aller Regel lebenslange Behandlung der Narkolepsie umfasst sowohl nichtmedikamentöse Strategien als auch die symptomatische medikamentöse Behandlung.

\section{ABSTRACT}

Narcolepsy is a hypersomnolence disorder of central origin that presents with a disturbance of the wake-sleep regulation. Lead symptoms consist of excessive daytime sleepiness and cataplexy. Nowadays, two types of narcolepsy are distinguished. Type 1 narcolepsy, formerly known as narcolepsy with cataplexy, is based on hypocretin deficiency. The cause of type 2 narcolepsy, formerly known as narcolepsy without cataplexy, remains mainly unknown. A multimodal approach is necessary for diagnosis. The mean latency between the onset of disease and diagnosis in Europe ranges 14 years. Narcolepsy has a major impact on workability and quality of life. The management of narcolepsy is usually life-long and includes non-pharmacological approaches and a symptomatic pharmacological treatment.

\section{Einleitung}

Narkolepsie ist eine seltene chronische neurologische Erkrankung, die mit einer Störung der Wach-Schlaf-Regulation einhergeht.

Die klinischen Leitsymptome sind eine exzessive Tagesschläfrigkeit, Kataplexien, hypnagogene/hypnopompe Halluzinationen,
Schlaflähmungen und ein fragmentierter Nachtschlaf, wobei nur bei einem Drittel der Patienten alle Leitsymptome vorhanden sind [1]. Neben den Leitsymptomen bestehen verschiedene weitere assoziierte Symptome, welche unten aufgeführt sind. 
Die fehlende Spezifität vieler Untersuchungsergebnisse erfordert einen multimodalen Diagnoseansatz. Die Tatsache, dass die Latenz zwischen Erkrankungsbeginn und Diagnosestellung in Europa momentan im Mittel 14 Jahre beträgt [2], illustriert die bestehenden Schwierigkeiten. Zuletzt konnten wichtige Erkenntnisse zur Pathophysiologie der Erkrankung gewonnen werden. Der Nachweis, dass spezifische T-Zellen beim Untergang Hypocretin-sezernierender Zellen eine entscheidende Rolle spielen, stützt die Hypothese einer autoimmunen Ätiologie [3, 4]. Die erfreuliche dynamische Entwicklung der Therapiemöglichkeiten mit Zulassung verschiedener neuer Medikamente in den letzten Jahren zeigt, dass eine frühzeitige Diagnosestellung therapeutische Relevanz hat. Dieser Artikel gibt eine aktuelle Übersicht über das Erkrankungsbild und seine diagnostischen und therapeutischen Möglichkeiten.

\section{Klinisches Bild}

\section{Definition}

Aktuell unterteilt man gemäß der International Classification of Sleep Disorders (ICSD-3) die Narkolepsie in Typ 1 (NT1), früher Narkolepsie mit Kataplexie, und Typ 2 (NT2), früher Narkolepsie ohne Kataplexie (siehe $>$ Abb. 1 [5]).

\section{Symptome}

\section{Tagesschläfrigkeit}

Meist ist die exzessive Tagesschläfrigkeit das erste und am stärksten beeinträchtigende Symptom. Zum Symptom der exzessiven Tagesschläfrigkeit, die chronisch vorliegt, gehören auch imperative Einschlafattacken, automatische Handlungen (als Ausdruck von Mikroschlaf) und ein erhöhtes Schlafbedürfnis.
Die Tagesschläfrigkeit ist verstärkt bei monotonen Tätigkeiten. In $80 \%$ der Fälle kommt es zu imperativen Schlafattacken von 15-20 Minuten [6], die im Gegensatz zu Patienten mit idiopathischer Hypersomnie als erfrischend wahrgenommen werden. Hinweisend auf eine Narkolepsie sind zudem Träume während dieser Tagschlafphasen, welche bei Gesunden in der Regel nicht vorkommen. Relevant ist die Unterscheidung zwischen exzessiver Tagesschläfrigkeit und Müdigkeit oder auch Fatigue, die konkomittant auftreten kann und therapeutisch oft nicht gut anspricht [7].

\section{Kataplexien}

Kataplexien sind ein pathognomonisches Symptom der NT1. Hierbei kommt es zu einem meist bilateral-symmetrischen plötzlichen Verlust des Muskeltonus, oft beginnend im Gesicht/Hals mit Übergang zu den Extremitäten, die Atemmuskulatur ist immer ausgespart. Bei sehr kurzen Attacken ist ein Sturz nicht obligat, die Patienten berichten dann oft über „weiche Knie“. Das Bewusstsein bleibt erhalten. Auslöser sind oftmals starke, sehr oft positive Emotionen (fast immer Lachen). Während einer Kataplexie-Episode, die üblicherweise wenige Sekunden bis wenige Minuten andauert, besteht, falls untersuchbar, eine Areflexie. Kataplexien sind zeitlich oft das zweite Symptom und können erst Jahre nach Beginn der exzessiven Tagesschläfrigkeit auftreten [7].

\section{Halluzinationen}

Diese treten häufig beim Einschlafen (hypnagog) und selten beim Erwachen (hypnopomp) auf, haben oft einen ängstigenden Charakter (z.B. Gefühl der Anwesenheit von Menschen oder Tieren im Zimmer) [1]. Im Gegensatz zu Psychosen sind sich Menschen mit Narkolepsie der Irrealität der Halluzinationen bewusst. Halluzinationen können sowohl visuell, akustisch als auch haptisch/ taktil sein.

\footnotetext{
Narkolepsie Typ 1 (NT1):

Kriterien a) und b) müssen erfüllt sein:

a) Der Patient hat tägliche Episoden von chronischer Tagesschläfrigkeit, nicht unterdrückbarem Schlafbedürfnis oder Schlafattacken über Tage für mindestens 3 Monate.

b) Das Vorhandensein von mindestens 1 der folgenden:

i. Kataplexie und eine mittlere Einschlaflatenz von $\leq 8$ Minuten und $\geq 2$ SOREMP im standardisierten MSLT. SOREMP (innerhalb von 15 Minuten nach Schlafbeginn) in der PSG in der Nacht vor MSLT kann einen der SOREMP im MSLT ersetzen.

ii. Hypocretin-Konzentration im Liquor ist entweder $<110 \mathrm{pg} / \mathrm{mL}$ oder $<1 / 3$ der Mittelwerte bei gesunden Probanden mit demselben standardisierten Assay*

Narkolepsie Typ 2 (NT2):

Kriterien a)-e) müssen erfüllt sein:

a) Der Patient hat tägliche Episoden von chronischer Tagesschläfrigkeit, nicht unterdrückbarem Schlafbedürfnis oder Schlafattacken über Tage für mindestens 3 Monate

b) eine mittlere Einschlaflatenz von $\leq 8$ Minuten und $\geq 2$ SOREMP im standardisierten MSLT. SOREMP (innerhalb von 15 Minuten nach Schlafbeginn) in der PSG in der Nacht vor MSLT kann einen der SOREMP im MSLT ersetzen.

c) Keine Kataplexie.

d) Die Hypocretin-1 Konzentration im Liquor wurde entweder nicht gemessen oder die gemessene Konzentration ist entweder $>110 \mathrm{pg} / \mathrm{mL}$ oder $>1 / 3$ der Mittelwerte bei gesunden Probanden mit demselben standardisierten Assay*.

e) Die exzessive Tagesschläfrigkeit und/oder MSLT Ergebnisse sind nicht durch andere Gründe erklärbar, wie Schlafdefizit, obstruktive Schlafapnoe, verzögertes Schlafphasensyndrom, Medikamenteneinnahme- oder Medikamentenabsetzprobleme
}

SOREMP: sleep onset REM period; MSLT: Multipler Schlaflatenztest; MSLT: Multipler Schlaflatenz Test; PSG: Polysomnographie;

* Es ist unbedingt darauf zu achten, dass eine validierte Messmethode (RIA) angewandt wird. 


\section{Schlaflähmung}

Bei Schlaflähmungen kommt es beim Erwachen zu einer kurzen, 1-2 Minuten andauernden Unfähigkeit zu sprechen oder sich zu bewegen im Übergang vom Wachzustand zu Schlaf (oder umgekehrt). Diese können auch bei Gesunden auftreten, dann oft seltener ( $\leq 1$-mal jährlich) als bei Menschen mit Narkolepsie [1].

\section{Fragmentierter Nachtschlaf}

Menschen mit Narkolepsie berichten oft eine schlechte Schlafqualität mit häufigem nächtlichen Erwachen und Schwierigkeiten, wieder einzuschlafen, was sich auch polysomnographisch belegen lässt. Das Ausmaß der nächtlichen Schlaffragmentierung korreliert nur schwach mit dem Ausmaß der Tagesschläfrigkeit [8].

\section{Assoziierte Symptome}

Menschen mit Narkolepsie haben ein erhöhtes Risiko für weitere Symptome und Erkrankungen, die zusätzlich behandlungsbedürftig sein können. Hierzu gehören weitere Schlafstörungen wie obstruktive Schlafapnoe, Restless-legs-Syndrom, REM-Schlafverhaltensstörungen und Somnambulismus sowie Adipositas, aber auch psychiatrische Erkrankungen, v.a. Angststörungen und Depressionen [8-11].

\section{Epidemiologie}

Die Prävalenz für die Narkolepsie insgesamt beträgt in Europa und Nordamerika etwa 25-79/100.000 Einwohner [12-14]. Bisherige epidemiologische Studien differenzieren in Narkolepsie mit Kataplexie, entsprechend NT1, und ohne Kataplexie, a.e. NT2 entsprechend. Für Narkolepsie mit Kataplexie sind Prävalenzraten von 14-36/100.000 Einwohner beschrieben [12, 14]. Eine aktuelle epidemiologische Arbeit ergab für die Narkolepsie ohne Kataplexie Prävalenzraten von 65/100.000 Einwohnern [14]. In Deutschland werden Inzidenzraten von 0,6-1/100.000 Personenjahre angenommen [15]. Das Erkrankungsalter liegt meist am Ende des 2. und Beginn des 3. Lebensjahrzehnts, ein Beginn im Kindesalter und mittleren Erwachsenenalter ist aber möglich [16, 17]. Männer und Frauen sind in etwa gleich häufig betroffen [7].

\section{Ursachen}

\section{NT1}

Bei NT1 kommt es zu einem Untergang von Hypocretin-sezernierenden Neuronen, welche ausschließlich im lateralen Hypothalamus lokalisiert sind. Die Neuropeptide Hypocretin/Orexin wurden 1998 unabhängig von zwei Arbeitsgruppen entdeckt [18, 19]. Hieraus resultiert die synonyme Verwendung von Hypocretin bzw. Orexin, wobei in der deutschsprachigen Literatur vornehmlich die Bezeichnung Hypocretin verwendet wird. Hypocretin wird im Wachen sezerniert und erhöht die Aktivität in Hirnregionen, die der Erhaltung der Wachheit dienen, und verhindert einen inadäquaten Übergang in REM- und Non-REM-Schlaf [20]. Der Verlust von Hypocretin führt dazu, dass sich REM-Schlaf-assoziierte Phänomene in Wachheitsphasen hineindrängen. Die Ursache der Hypocretin-Defizienz ist unklar, ein autoimmuner
Prozess, der multifaktoriell durch genetische Prädisposition, Umweltfaktoren und provozierende Ereignisse beeinflusst ist, wird angenommen [3, 7]. In der Arbeit von Latorre, Kallweit et al. konnten erstmalig autoreaktive CD4 + und CD8 + Zellen gegen Hypocretin nachgewiesen werden [3, 7]. Ein autoimmuner Trigger wurde zudem angenommen, als die Narkolepsie-Inzidenzraten nach der Grippepandemie mit Influenza A H1N1-Virus und/ oder der nachfolgenden Massenimpfung mit dem Impfstoff Pandemrix 2009/2010 anstiegen. Eine aktuelle Studie bestätigte diese Hypothese, indem sie zeigte, dass die Zerstörung der Hypocretin-sezernierenden Zellen durch spezifische T-Zellen durch molekulares Mimikry mit H1N1 erfolgen könnte [4]. Bei Menschen mit NT1 wurde in > 90\% der Fälle HLA DQB1 ${ }^{*} 0602$ nachgewiesen [7], was auf eine genetische Prädisposition hinweist. Menschen mit diesem Haplotyp haben ein 250-fach erhöhtes Risiko, an NT1 zu erkranken [21]. Die Spezifität des Befundes ist hingegen gering, da HLA DQB1*0602 auch bei bis zu 35\% der Normalbevölkerung vorkommt [22]. Das Risiko, eine NT1 zu entwickeln, liegt für Angehörige 1. Grades bei 1-2\% [7] und ist damit 20-40\% höher als in der Gesamtpopulation [12-14]. Dass bei monozygoten Zwillingen nur in 25\% der Fälle beide erkranken [23], lässt den Einfluss weiterer Faktoren, welche zur Ausbildung der Erkrankung führen, vermuten.

\section{NT2}

Die Ursache der NT2 ist unbekannt. Ein messbarer HypocretinMangel liegt nicht vor [24]. Vermutlich handelt es sich hier nicht um eine einheitliche Entität. Die HLA DQB1*0602-Assoziation besteht nur bei 40\% der Fälle [25]. In manchen Fällen kann eine NT2 im Verlauf in eine NT1 übergehen [26], kann in diesen Fällen möglicherweise als deren Prodrom gesehen werden. Auch bei Patienten mit NT2 wurden autoreaktive T-Zellen gegen Hypocretin-produzierende Neurone gefunden [7].

\section{Sekundäre Narkolepsie}

Selten können Läsionen im Hypothalamus und Mittelhirn mit Schädigung Hypocretin-sezernierender Zellen oder deren Projektionsbahnen eine sekundäre Narkolepsie verursachen. Als Ursachen wurden Schlaganfälle, Tumore, vaskuläre Malformationen, Multiple Sklerose und andere autoimmune Entzündungen (Neurosarkoidose, anti-Ma2-Enzephalitis) gefunden [27, 28].

Auch bei den genetischen Syndromen Prader-Willi-Syndrom [29] oder Niemann-Pick Typ C [30] sind exzessive Tagesmüdigkeit und Kataplexie-artige Episoden beschrieben worden. Als wichtiges klinisches Merkmal bestehen bei sekundärer Narkolepsie zumeist auch fokal-neurologische Defizite, da nicht nur isoliert die Hypocretin-sezernierenden Neurone geschädigt sind.

\section{Diagnostik}

Die Diagnose wird gemäß den ICSD-3-Kriterien erstellt [5].

\section{Anamnese}

Eine umfangreiche Schlaf-Wach-Anamnese ist die Grundlage für die Diagnose. Nachfolgend sind hilfreiche Fragen bei Verdacht auf eine Narkolepsie aufgelistet, deren Implikationen in Klammern dahinter eingefügt sind (modifiziert nach [1]): 
- Sind Sie über die meiste Zeit des Tages schläfrig? (exzessive Tagesschläfrigkeit)

- Wie oft ist es in den letzten beiden Wochen passiert, dass Sie über Tage eingeschlafen sind, ohne es zu wollen? (Imperativer Schlafdrang mit Einschlafattacken)

- Sind kurze Schlafphasen über Tage hilfreich gegen die Schläfrigkeit? (erholsame Wirkung von kurzen Schlafpausen)

- Haben Sie lebhafte Träume, wenn Sie tagsüber schlafen? (mögliche REM-Schlafphasen über Tage)

- Kommt es vor, dass Sie während des Einschlafens Dinge hören, sehen oder fühlen, von denen Sie wissen, dass sie nicht da sind? (Hypnagoge Halluzinationen)

- Kommt es vor, dass Sie sich nach dem Erwachen für kurze Zeit nicht bewegen können? (Schlaflähmung)

- Kann es passieren, dass Sie eine akute Muskelschwäche haben, wenn sie plötzlich lachen müssen? (Kataplexien)

Zusätzliche Symptome und Begleiterkrankungen, die unter 1.2.5 aufgeführt sind, sollten in der Anamnese gezielt erfragt werden. Differenzialdiagnosen zu den Leitsymptomen sind in

- Tab. 1 dargestellt.

\section{Multipler Schlaflatenztest (MSLT)}

Der MSLT ist integraler Bestandteil zur Diagnosesicherung bei Verdacht auf Narkolepsie. Erfasst werden die Einschlaflatenz und das Auftreten von umittelbaren REM-Schlaf-Phasen (sleep onset
REM-Phasen, SOREMP) nach Schlafbeginn. Aufgrund seiner oft entscheidenden Aussage sollte er nur unter standardisierten Bedingungen von erfahrenen Untersuchern durchgeführt werden, entsprechende Konsensus-Empfehlungen finden sich in [31]. Eine mittlere Einschlaflatenz unter 8 Minuten und der Nachweis von $\geq 2$ SOREMP bei 5 Einschlaftests sind hinweisend auf eine Narkolepsie [32]. In der Nacht zuvor soll eine Polysomnographie erfolgen. Diese dient u.a. dazu, die Schlafzeit vor dem MSLT zu beurteilen, die nicht unter 6 Stunden liegen sollte. Zudem kann in der Beurteilung ein SOREMP in der Polysomnographie, der innerhalb von 15 Minuten nach Schlafbeginn auftritt, einen SOREMP in den Einschlaftests ersetzen [5]. Sowohl eine verminderte Einschlaflatenz als auch SOREMP sind nicht pathognomonisch für eine Narkolepsie, sondern können auch bei sonstigen Störungen im Tag-Wach-Rhythmus auftreten, z.B. bei Schichtarbeitern, obstruktiver Schlafapnoe und sonstigem Schlafmangel [26]. Auch kann die Einnahme REM-Schlaf-unterdrückender Medikamente, z.B. Antidepressiva und Sedativa, SOREMP unterdrücken oder nach deren Absetzen vermehrt erzeugen. Zudem haben bis zu 20\% der Normalbevölkerung eine mittlere Einschlaflatenz $\leq 8$ Minuten [31], und bei 5-10\% der Normalbevölkerung können $\geq 2$ SOREMP im MSLT auftreten [31, 33]. Der MSLT kann daher in 20-30\% der Fälle falsch-negativ oder falsch-positiv sein [34, 35]. Bei negativem Testergebnis und weiterhin bestehendem starken Verdacht auf eine Narkolepsie sollte er daher wiederholt werden.

-Tab. 1 Differenzialdiagnosen von Symptomen bei Narkolepsie.

\begin{tabular}{|c|c|}
\hline Symptom & Differenzialdiagnosen \\
\hline $\begin{array}{l}\text { Exzessive } \\
\text { Tagesschläfrigkeit }\end{array}$ & $\begin{array}{l}\text { Schlafmangel, Störungen im zirkadianen Wach-Schlaf-Rhythmus (Schichtarbeit, Jetlag) } \\
\text { Obstruktive Schlafapnoe, zentrale Schlafapnoe, Schlaf-assoziierte Hypoventilation oder Hypoxämie } \\
\text { Zentrale Hypersomnolenzen } \\
\text { " Idiopathische Hypersomnie } \\
\text { " Kleine-Levin-Syndrom } \\
\text { Andere neurologische Erkrankungen (Parkinson, Demenzen, Multiple Sklerose, Amyotrophe Lateralsklerose, } \\
\text { Thalamusläsionen) } \\
\text { Metabolisch/Genetisch (Hypothyreose, Nieren-, Leber, Nebennieren-Insuffizienz, Übergewicht, Niemann-Pick Typ C, } \\
\text { Prader-Willi-Syndrom) } \\
\text { Psychiatrische Erkrankungen (Depression, Angststörung, Substanzabusus, psychogene Schläfrigkeit) } \\
\text { Medikamente/Drogen (Benzodiazepine, Sedativa, Antipsychotika, Opioide, Betablocker, Barbiturate, Antikonvulsiva, Anti- } \\
\text { depressiva, Antihistaminika) }\end{array}$ \\
\hline Kataplexie & $\begin{array}{l}\text { Konversionsstörungen (ggf. Versuch der Videodokumentation mit Aufmerksamkeit auf Gesicht und Hals können manch- } \\
\text { mal diagnostisch hilfreich sein, in der Kataplexie besteht Areflexie) } \\
\text { Drop attacks } \\
\text { Synkopen/Präsynkopen } \\
\text { Epilepsie: Juvenile Myoklonische Epilepsie (partielle Kataplexien), gelastische Anfälle }\end{array}$ \\
\hline $\begin{array}{l}\text { Hypnagoge/hypnopompe } \\
\text { Halluzinationen und } \\
\text { Schlaflähmung }\end{array}$ & $\begin{array}{l}\text { Schlafmangel } \\
\text { Störungen im zirkadianen Wach-Schlaf-Rhythmus (Schichtarbeit, Jetlag) } \\
\text { Obstruktive Schlafapnoe } \\
\text { Angststörung } \\
\text { Beendigung REM-Schlaf-unterdrückender Medikamente/Drogen (Alkohol, Amphetamine, Antidepressiva) } \\
\text { Schizophrenie (Narkolepsie-Patienten erkennen die Halluzinationen oft als traumähnliche Phänomene ohne psychotisches } \\
\text { Erleben) } \\
\text { Physiologisch bei 20\% der gesunden Normalbevölkerung (1-2x/Jahr, bei Narkolepsie mit etwa 2-3x/Monat häufiger) }\end{array}$ \\
\hline
\end{tabular}




\section{Multipler Wachbleibetest (engl. Maintenance of Wakefulness Test, MWT)}

Der MWT misst die Fähigkeit des Probanden, in einer monotonen Situation für eine festgelegte Zeitspanne wach zu bleiben. Evaluiert wird die Einschlaflatenz. Empfohlen sind 4 Wachtests zu je 40 Minuten im Abstand von 2 Stunden (standardisierte Empfehlungen in [31]). Der MWT wird in der Regel zur Evaluation des Therapieansprechens bei exzessiver Tagesschläfrigkeit und bei verkehrsgutachterlichen Fragestellungen durchgeführt. Zur Diagnosestellung der Narkolepsie findet der MWT keine Berücksichtigung.

\section{Hypocretin im Liquor}

Die Bestimmung von Hypocretin im Liquor mittels Radioimmunoassay (RIA) hat sich in den letzten Jahren zwar zunehmend etabliert, der Test ist allerdings nur zu wissenschaftlichen Fragestellungen zugelassen. Bei nicht validierten Methoden kommt es wiederholt zu falsch-positiven Werten (erniedrigte Hypocretinwerte), die dann zu einer falschen Diagnose führen können. Zur Bestimmung von Hypocretin sollen PolypropylenProbenröhrchen verwendet werden, damit eine Adsorption an der Gefäßwand bei Polystyrol oder Glas vermieden wird und somit keine falsch-niedrigen Werte erhoben werden. HypocretinWerte unterhalb von $110 \mathrm{pg} / \mathrm{ml}$ im Liquor gelten als pathologisch und werden richtungsweisend für das Vorliegen einer Narkolepsie angesehen. Messwerte zwischen 110-200 pg/ml werden als „intermediärer Bereich“ bezeichnet, und Werte, die höher als 200 pg/ml liegen, werden als normal erachtet [24, 36]. Der Test ist sinnvoll, wenn die Diagnose anhand anderer Kriterien (s. - Abb. 1) nicht gesichert werden kann, jedoch der hochgradige Verdacht auf das Vorliegen einer Narkolepsie fortbesteht. Die Diagnosestellung anhand der alleinigen Bestimmung des Hypocretin-Wertes ohne schlafmedizinische Diagnostik (v.a. MSLT) ist, auch aus o.g. Gründen, nicht empfehlenswert.

\section{HLA-Bestimmung}

Die Bestimmung von HLA DQB1 0602 wird aktuell nicht zur Routine-Testung empfohlen und ist kein Diagnosekriterium in der aktuellen Definition (s. > Abb. 1). Sie kann jedoch für die Beratung wichtig sein, da Menschen mit diesem Haplotyp ein 250fach erhöhtes Risiko haben, an Narkolepsie zu erkranken. Der fehlende Nachweis macht die Verdachtsdiagnose einer NT1 unwahrscheinlicher [7].

\section{Schlaffragebögen}

Die Epworth Sleepiness Scale (ESS) ist ein in deutscher Übersetzung erhältlicher 8-Item-Kurzfragebogen zur Erfassung der Tagesschläfrigkeit. Er kann als Screeningtest in der Diagnostik und als Therapiekontrollinstrument verwendet werden. Ein ebenfalls gut validierter Screeningtest für NT1 ist die Swiss Narcolepsy Scale [37] (https://www.swissnarcolepsyscale.com). Zudem können Schlaftagebücher zur Langzeitbeurteilung sinnvoll sein. Darin wird in einem Abend- und einem Morgenprotokoll zweimal täglich für die Dauer von ca. 2 Wochen die eigene subjektive Einschätzung zu Schlaf und Tagesmüdigkeit dokumentiert. Hieraus können Rückschlüsse auf den persönlichen Schlafrhythmus gezogen und eine individuelle medikamentöse Therapie angepasst werden. Auch für Kataplexien sollte ein Tagebuch geführt werden, welche die Häufigkeit und ggf. Auslöser dokumentiert (erhältlich zum Beispiel unter: www.dgsm.de).

\section{Bildgebung}

Eine cerebrale Bildgebung ist nicht zwingend gefordert, sollte aber zum Ausschluss von anderen Ursachen für Hypersomnolenz durchgeführt werden [1].

\section{Therapie}

Die Therapie bei Narkolepsie ist eine symptomatische Dauertherapie. Primäre Zielsymptome sind die Besserung der exzessiven Tagesschläfrigkeit und der REM-Schlaf-assoziierten Beschwerden, in erster Linie der Kataplexien.

\section{Allgemeine und nichtmedikamentöse Therapie}

Die nichtmedikamentöse Behandlung umfasst Verhaltensempfehlungen wie regelmäßiges Einhalten der individuell notwendigen Schlafmenge, das Einlegen eines kurzen Tagschlafs von 1520 Minuten zur temporären Verbesserung der Wachheit. Weiterhin werden eine ausgeglichene Ernährung, der Genuss stimulierender Getränke bei Alkohol- und Nikotinkarenz und körperliches Training empfohlen. Die Einübung von spezifischen Bewältigungsmustern, die Beratung bei der Auswahl des Arbeitsplatzes und die Organisation der Arbeitsabläufe dienen der Minderung von sozialen Nachteilen. Menschen mit Narkolepsie haben ein erhöhtes Risiko, Autounfälle zu verursachen [38], weshalb bei bestehender Symptomatik (und ohne Behandlung) keine Eignung zum Führen eines Kraftfahrzeuges besteht, worüber der Arzt ausführlich aufklären muss. Bei erfolgreicher Therapie sollte eine verkehrsmedizinische Begutachtung zur Beurteilung der Fahreignung erfolgen [39].

\section{Medikamentöse Therapie}

In der Regel sind die oben genannten allgemeinen Therapieempfehlungen nicht ausreichend, sodass eine medikamentöse Dauertherapie zusätzlich nötig ist [40, 41]. Die vorhandenen Medikamente wirken entweder auf eines oder auf mehrere Leitsymptome. Alle Substanzen sind nur für Erwachsene zugelassen und werden in der Schwangerschaft und Stillzeit nicht empfohlen. Aufgrund des Missbrauchspotenzials sind einige Medikamente nach dem Betäubungsmittelgesetz (BTM) zu verordnen. Die aktuell verfügbaren Substanzen sind im Detail in $>$ Tab. 2 dargestellt.

\section{Medikamente gegen exzessive Tagesschläfrigkeit}

Modafinil wird seit über 20 Jahren zur Behandlung der exzessiven Tagesschläfrigkeit eingesetzt. Die Wirksamkeit wurde in zwei randomisierten Studien nachgewiesen [42, 43]. Als Wirkmechanismus wird eine Dopamin-Wiederaufnahmehemmung angenommen [41]. Es wird im Allgemeinen gut vertragen und hat ein geringes Abhängigkeitspotenzial. Es bestehen Warnhinweise zu erhöhten Fehlbildungsraten unter Therapie, wobei kein einheitliches Fehlbildungsmuster zu erkennen ist. Aufgrund der Cytochrom-P450-Enzyminduktion ist die Wirkung von hormonellen Kontrazeptiva vermindert, weshalb sichere alternative 

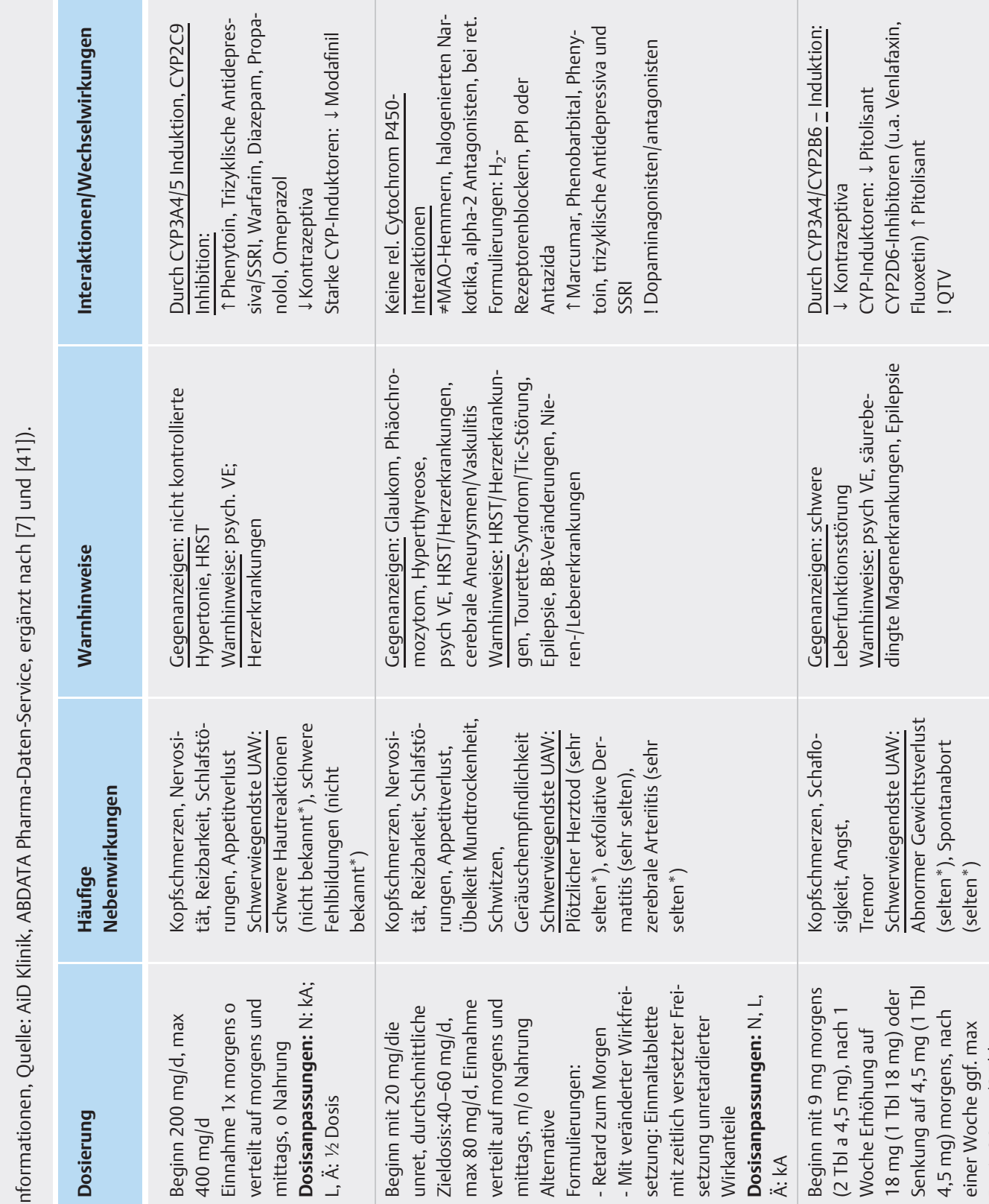

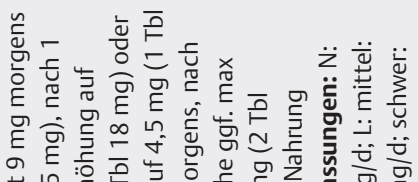

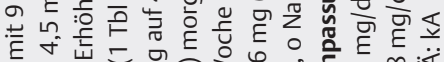

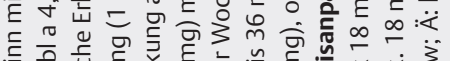

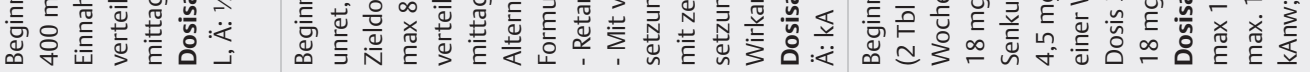
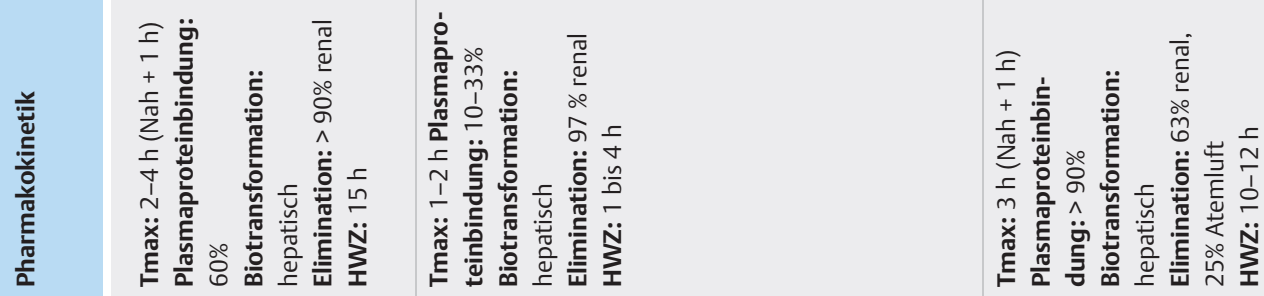


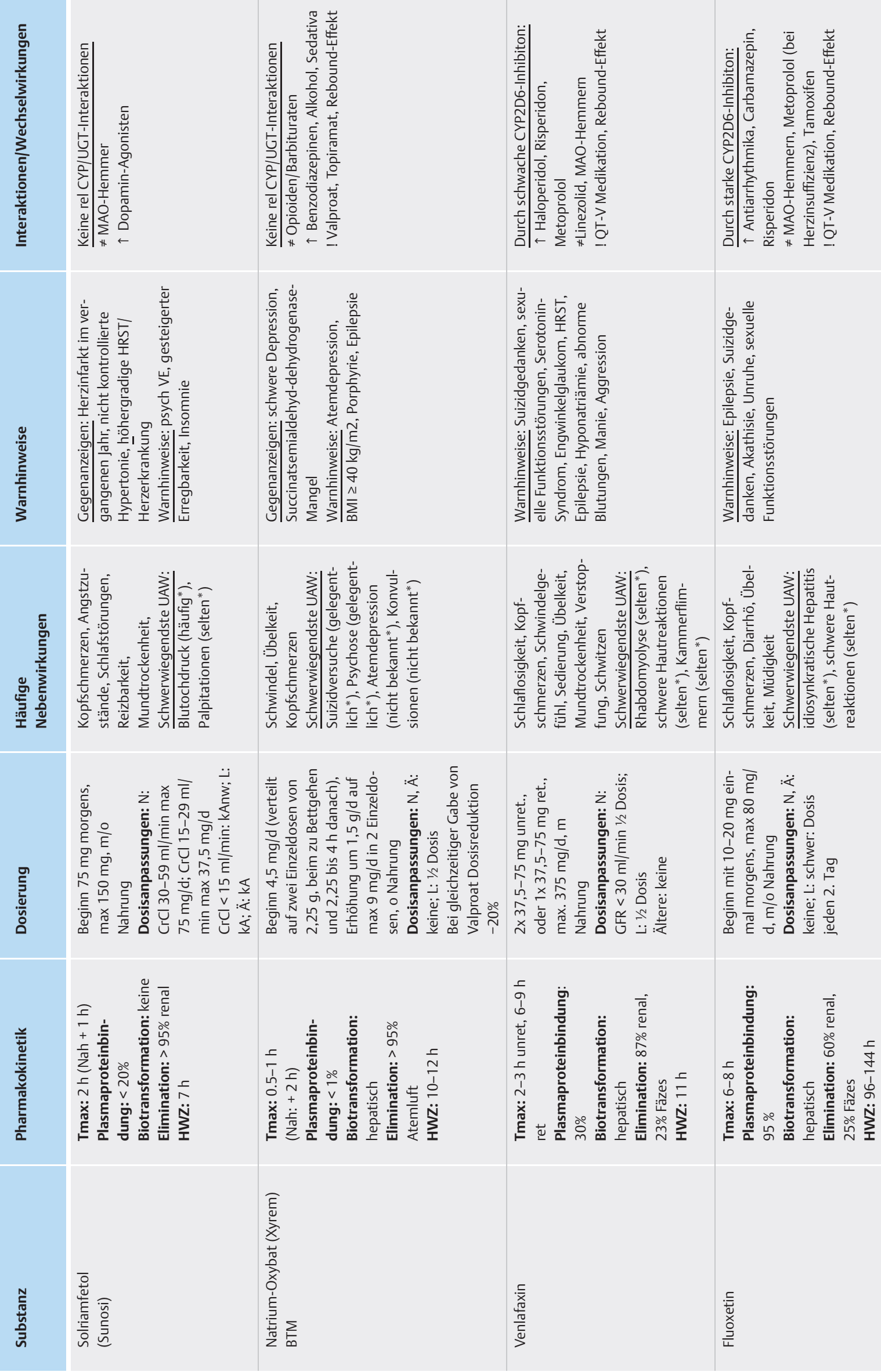



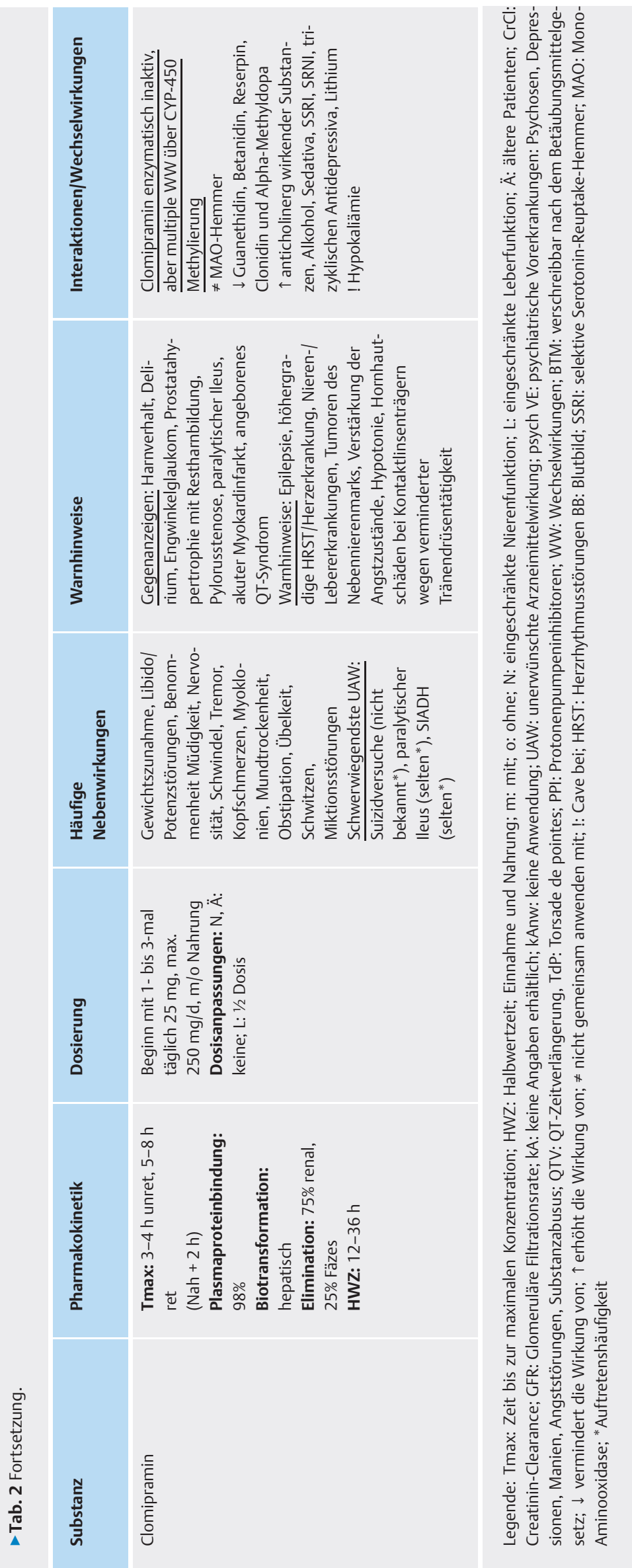
Verhütungsmethoden gewählt werden müssen. Armodafinil, das aktive R-Enantiomer mit verlängerter Wirkung, ist als Medikament nur in den USA zugelassen.

Methylphenidat ist ein Dopamin- und Noradrenalin-Wiederaufnahmehemmer mit guter Wirkung auf die exzessive Tagesschläfrigkeit. Das Nebenwirkungsprofil ist im Vergleich zu Modafinil ungünstiger, weshalb Methylphenidat heute eher zweite Wahl ist. Es sind verschiedene Formulierungen erhältlich. Initial kommen zunächst unretardierte Formulierungen zum Einsatz. Im Verlauf kann zur Vermeidung von Missbrauchs- und Gewöhnungseffekten auf retardierte Präparate oder Präparate mit veränderter Wirkstofffreisetzung umgestellt werden. Bei Letzteren kommt es zur zeitversetzten prozentualen Freigabe des Wirkstoffes in unretardierter Form. Auch bei den retardierten Formulierungen kann bei ausgeprägter Tagesmüdigkeit am Nachmittag eine zweite Gabe am frühen Nachmittag (vor 14 Uhr) hilfreich sein.

Solriamfetol (Sunosi) wurde Anfang 2020 in Europa zur Behandlung exzessiver Tagesschläfrigkeit bei Narkolepsie und bei obstruktiver Schlafapnoe zugelassen. Es ist seit Mai 2020 in Deutschland verfügbar und wird für die Indikation Narkolepsie von den gesetzlichen Krankenkassen erstattet. Der Wirkmechanismus besteht in einer Dopamin- und Noradrenalin-Wiederaufnahmehemmung [44].

\section{Medikamente gegen exzessive Tagesschläfrigkeit und Kataplexien}

Pitolisant (Wakix) wirkt gegen exzessive Tagesschläfrigkeit und Kataplexien, geringer auch gegen Halluzinationen und Schlaflähmungen. Der Histamin-H3-Rezeptor-Antagonist zeigte in den Zulassungsstudien eine Überlegenheit gegenüber Plazebo bei vergleichbarer Wirkung zu Modafinil [45, 46]. Wegen einer CYP3A4-Induktion kann die Wirkung hormoneller Kontrazeptiva reduziert werden, weshalb alternative Verhütungsmethoden bei Frauen im gebärfähigen Alter nötig sind. CYP2D6-Inhibitoren (u. a. Venlafaxin) wiederum können die Pitolisant-Konzentration erhöhen, weshalb auf Nebenwirkungen zu achten ist und Dosisanpassungen bedacht werden sollten.

Natrium-Oxybat (Xyrem), das Natriumsalz der Gammahydroxybuttersäure (GHB), einem Metaboliten der Gamma-Amino-Buttersäure (GABA), wirkt gegen fragmentierten Nachtschlaf, wodurch die Tagesmüdigkeit verbessert wird, und gegen Kataplexien [47-50]. Der genaue Wirkmechanismus ist nicht bekannt, man nimmt eine GABAerge Wirkung mit Unterdrückung von REM-Schlaf und Förderung tiefer Schlafphasen an. Es wird in flüssiger Form in zwei geteilten Dosierungen eingenommen, beim Zubettgehen und 2,5-4 Stunden danach. Dies erfordert in der ersten Zeit das Stellen eines Weckers, im Verlauf kommt es meist zu einer Konditionierung mit selbstständigem Erwachen des Patienten zum zweiten Einnahmezeitpunkt. Praktisch empfiehlt es sich, dass beide Dosierungen bereits vor dem Zubettgehen zubereitet und auf den Nachttisch gestellt werden. Eine wichtige Interaktion besteht mit Valproat, das als GHB-DehydrogenaseInhibitor zu einer Wirkverstärkung führen kann, weshalb in dieser Kombination eine Reduktion von Natrium-Oxybat um 20\% empfohlen wird. GHB besitzt ein relevantes Missbrauchspotenzial und kann z.B. als Inhalt von KO-Tropfen verwendet werden.

\section{Medikamente gegen Kataplexien}

Venlafaxin wirkt gut gegen Kataplexien, wenngleich die Empfehlungen auf Expertenkonsens und nicht auf klinischen Studien beruhen [51], weshalb es nicht zur Behandlung der Narkolepsie zugelassen ist. Venlafaxin ist ein Serotonin- und Noradrenalinwiederaufnahme-Inhibitor (SNRI). Die Wirkung setzt bei Narkolepsie oft schon in geringen Dosierungen und innerhalb weniger Tage an. Nach abruptem Absetzen sind Rebound-Phänomene mit erhöhter Kataplexierate möglich, weshalb eine schrittweise Abdosierung empfohlen wird.

Clomipramin ist als einziges Antidepressivum und nur in Deutschland zur Behandlung von Kataplexien bei Narkolepsie zugelassen. Aufgrund des ungünstigeren Nebenwirkungs- und hohen Interaktionspotenzials wird es nicht zur Erstlinien-Therapie empfohlen. Wegen der Metabolisierung über verschiedene CYP450-Isoenzyme (v.a. CYP3A4, CYP2C19, CYP1A2, CYP2D6) bestehen multiple Wechselwirkungen, wenngleich Clomipramin selbst nicht enzymatisch aktiv. Eine Interaktion zu hormonellen Kontrazeptiva besteht nicht.

Fluoxetin, ein Serotonin-Wiederaufnahmehemmer, wirkt ebenfalls gegen Kataplexien, die Empfehlung basiert auf Expertenkonsens, es besteht keine Zulassung für Narkolepsie.

\section{Auswahl der Medikamente}

Die Auswahl der Medikamente erfolgt individuell nach den bestehenden Beschwerden und nach dem individuellen Risikoprofil. Eine Übersicht hierüber gibt $>$ Tab. 3. Oft ist es zudem nötig, verschiedene Medikamente zu kombinieren. Die Substanzen zeigen untereinander nur wenige relevante Interaktionen. Relevant ist, dass die Wirkung von Clomipramin bei Zugabe von Modafinil erhöht sein kann. Verschiedene relevante Interaktionen bestehen bei Pitolisant: Venlafaxin und Fluoxetin können die Wirkung verstärken, wohingegen Clomipramin die Wirkung vermindern kann. Nicht sinnvoll ist aus pharmakologischer Sicht eine Kombination von Modafinil mit Methylphenidat oder Solriamfetol.

\section{Take Home Message}

Narkolepsie ist eine chronische Erkrankung mit Störungen der Schlaf-Wach-Regulation. Ursächlich besteht bei NT1 eine Hypocretin-Defizienz, die Ursache der NT2 ist überwiegend unklar. In nahezu allen Fällen ist eine dauerhafte medikamentöse Therapie nötig, welche nach den bestehenden Symptomen und dem individuellen Verträglichkeitsprofil ausgewählt wird. Pitolisant (für Tagesschläfrigkeit und Kataplexien) und Solriamfetol (für Tagesschläfrigkeit) haben aktuell die Therapiemöglichkeiten erweitert.

\section{Fazit für die Praxis:}

- Narkolepsie ist eine chronische Erkrankung mit Störungen der Schlaf-Wach-Regulation.

- Leitsymptome sind exzessive Tagesschläfrigkeit, Kataplexien, hypnagogene/hypnopompe Halluzinationen, Schlaflähmungen und gestörter Nachtschlaf. 
- Tab. 3 Medikamenteneinsatz bei verschiedenen Symptomen.

\begin{tabular}{|l|l|l|}
\hline Symptom & Verbessert durch* & Verschlechtert durch \\
\hline Leitsymptome & & \\
\hline Exzessive Tagesschläfrigkeit & Methylphenidat, Modafinil, Solriamfetol & $\begin{array}{l}\text { Alkohol, sedierende Antidepressiva, Anti- } \\
\text { Histaminika, Benzodiazepine, Neurolep- } \\
\text { tika, Opiate ... }\end{array}$ \\
\hline Kataplexie & Clomipramin, Na-Oxybat, Pitolisant, Venlafaxin, & $\begin{array}{l}\text { plötzliches Absetzen von Antidepressiva, } \\
\text { Prazosin }\end{array}$ \\
\hline Exzessive Tagesmüdigkeit und Kataplexie & Na-Oxybat, Pitolisant & \\
\hline Hypnagoge Halluzinationen & Na-Oxybat, Pitolisant, Venlafaxin & \\
\hline Schlafparalyse & Na-Oxybat, Pitolisant, Venlafaxin & $\begin{array}{l}\text { Methylphenidat, Modafinil, Pitolisant, Sol- } \\
\text { riamfetol, Venlafaxin }\end{array}$ \\
\hline Fragmentierter Nachtschlaf & Na-Oxybat, Zolpidem & \\
\hline$*$ Aufzählung in alphabetischer Reihenfolge & & \\
\hline
\end{tabular}

- Bei Auftreten von Kataplexien besteht eine Narkolepsie Typ 1 (NT1), bei deren Fehlen eine Narkolepsie Typ 2 (NT2).

- Ursache der NT 1 ist eine Hypocretin-Defizienz, die Ursache der NT2 ist unbekannt.

- In allen Fällen ist eine dauerhafte symptomatische medikamentöse Therapie indiziert.

- Pitolisant und Solriamfetol haben aktuell die Therapiemöglichkeiten erweitert.

\section{Interessenkonflikt}

MPM erhielt Honorare für Vortrags- und Beratertätigkeit von UCB und EISAI.

JN berichtet keine Interessenkonflikte.

AT berichtet keine Interessenkonflikte.

UK erhielt Honorare für Vortrags- und Beratertätigkeit von AOP Orphan Pharmaceuticals, Bioprojet Pharma, Harmony Biosciences, Jazz Pharma, Takeda Pharmaceutical, UCB Pharma. Die wissenschaftlichen Arbeiten wurden unterstützt durch das European Narcolepsy Network, die Universität Witten/Herdecke (interne Forschungsförderung Nr. 2020-31), Bioprojet Pharma, Harmony Biosciences und UCB Pharma.

\section{Literatur}

[1] Scammel TE Clinical features and diagnosis of narcolepsy in adults. wwwuptodatecom. UpToDate, Wolters Kluwer: Wolters Kluwer, 2020

[2] Luca G, Haba-Rubio ], Dauvilliers Y et al. Clinical, polysomnographic and genome-wide association analyses of narcolepsy with cataplexy: A European Narcolepsy Network study. J Sleep Res 2013; 22: 482-495

[3] Latorre D, Kallweit U, Armentani E et al. T cells in patients with narcolepsy target self-antigens of hypocretin neurons. Nature 2018; 562: 6368

[4] Luo G, Ambati A, Lin L et al. Autoimmunity to hypocretin and molecular mimicry to flu in type 1 narcolepsy. Proc Natl Acad Sci U S A 2018; 115: E12323-E12332
[5] Ruoff C, Rye D. The ICSD-3 and DSM-5 guidelines for diagnosing narcolepsy: Clinical relevance and practicality. Curr Med Res Opin 2016; 32: 1611-1622

[6] Khatami R, Luca G, Baumann CR et al. The European Narcolepsy Network (EU-NN) database. J Sleep Res 2016; 25: 356-364

[7] Bassetti CLA, Adamantidis A, Burdakov D et al. Narcolepsy - clinical spectrum, aetiopathophysiology, diagnosis and treatment. Nat Rev Neurol 2019; 15: 519-539

[8] Roth T, Dauvilliers Y, Mignot E et al. Disrupted nighttime sleep in narcolepsy. J Clin Sleep Med 2013; 9: 955-965

[9] Pizza F, Tartarotti S, Poryazova R et al. Sleep-disordered breathing and periodic limb movements in narcolepsy with cataplexy: A systematic analysis of 35 consecutive patients. Eur Neurol 2013; 70: 22-26

[10] Cohen A, Mandrekar J, St Louis EK et al. Comorbidities in a community sample of narcolepsy. Sleep Med 2018; 43: 14-18

[11] Ruoff CM, Reaven NL, Funk SE et al. High rates of psychiatric comorbidity in narcolepsy: findings from the Burden of Narcolepsy Disease (BOND) study of 9,312 patients in the United States. J Clin Psychiatry 2017; 78: 171-176

[12] Hublin C, Kaprio J, Partinen M et al. The prevalence of narcolepsy: An epidemiological study of the Finnish Twin Cohort. Ann Neurol 1994; 35 : 709-716

[13] Silber MH, Krahn LE, Olson EJ et al. The epidemiology of narcolepsy in Olmsted County, Minnesota: A population-based study. Sleep 2002; 25: 197-202

[14] Scheer D, Schwartz SW, Parr M et al. Prevalence and incidence of narcolepsy in a US health care claims database, 2008-2010. Sleep 2019; 42

[15] Oberle D, Drechsel-Bäuerle U, Schmidtmann I et al. Incidence of Narcolepsy in Germany. Sleep 2015; 38: 1619-1628

[16] Dauvilliers Y, Montplaisir J, Molinari $\mathrm{N}$ et al. Age at onset of narcolepsy in two large populations of patients in France and Quebec. Neurology 2001; 57: 2029-2033

[17] Rocca FL, Pizza F, Ricci E et al. Narcolepsy during childhood: an update. Neuropediatrics 2015; 46: 181-198

[18] Sakurai T, Amemiya A, Ishii M et al. Orexins and orexin receptors: a family of hypothalamic neuropeptides and $G$ protein-coupled receptors that regulate feeding behavior. Cell 1998; 92: 1 page following 696 
[19] de Lecea L, Kilduff TS, Peyron C et al. The hypocretins: Hypothalamusspecific peptides with neuroexcitatory activity. Proc Natl Acad Sci U S A 1998; 95: 322-327

[20] Scammell TE, Arrigoni E, Lipton JO. Neural Circuitry of Wakefulness and Sleep. Neuron 2017; 93: 747-765

[21] Tafti M, Hor H, Dauvilliers Y et al. DQB1 locus alone explains most of the risk and protection in narcolepsy with cataplexy in Europe. Sleep 2014; 37: 19-25

[22] Mignot E, Thorsby E. Narcolepsy and the HLA system. N Engl J Med 2001; 344: 692

[23] Mignot E. Genetic and familial aspects of narcolepsy. Neurology 1998; 50: $\mathrm{S} 16-22$

[24] Mignot E, Lammers G], Ripley B et al. The role of cerebrospinal fluid hypocretin measurement in the diagnosis of narcolepsy and other hypersomnias. Arch Neurol 2002; 59: 1553-1562

[25] Lin L, Hungs M, Mignot E. Narcolepsy and the HLA region. J Neuroimmunol 2001; 117: 9-20

[26] Baumann CR, Mignot E, Lammers G] et al. Challenges in diagnosing narcolepsy without cataplexy: a consensus statement. Sleep 2014; 37 : 1035-1042

[27] Nishino S, Kanbayashi T. Symptomatic narcolepsy, cataplexy and hypersomnia, and their implications in the hypothalamic hypocretin/orexin system. Sleep Med Rev 2005; 9: 269-310

[28] Kallweit U, Bassetti CLA, Oberholzer M et al. Coexisting narcolepsy (with and without cataplexy) and multiple sclerosis: six new cases and a literature review. J Neurol 2018; 265: 2071-2078

[29] Omokawa M, Ayabe T, Nagai T et al. Decline of CSF orexin (hypocretin) levels in Prader-Willi syndrome. Am J Med Genet A 2016; 170A: 11811186

[30] Nevsimalova S, Malinova V. Cataplexy and sleep disorders in NiemannPick type C disease. Curr Neurol Neurosci Rep 2015; 15: 522

[31] Littner MR, Kushida C, Wise M et al. Practice parameters for clinical use of the multiple sleep latency test and the maintenance of wakefulness test. Sleep 2005; 28: 113-121

[32] Sateia MJ. International classification of sleep disorders-third edition: Highlights and modifications. Chest 2014; 146: 1387-1394

[33] Mignot E, Lin L, Finn L et al. Correlates of sleep-onset REM periods during the Multiple Sleep Latency Test in community adults. Brain 2006; 129: 1609-1623

[34] Lopez R, Doukkali A, Barateau L et al. Test-retest reliability of the multiple sleep latency test in central disorders of hypersomnolence. Sleep 2017; 40

[35] Trotti LM, Staab BA, Rye DB. Test-retest reliability of the multiple sleep latency test in narcolepsy without cataplexy and idiopathic hypersomnia. J Clin Sleep Med 2013; 9: 789-795
[36] Ripley B, Overeem S, Fujiki N et al. CSF hypocretin/orexin levels in narcolepsy and other neurological conditions. Neurology 2001; 57: 22532258

[37] Bargiotas P, Dietmann A, Haynes AG et al. The Swiss Narcolepsy Scale (SNS) and its short form (sSNS) for the discrimination of narcolepsy in patients with hypersomnolence: A cohort study based on the Bern sleep-wake database. J Neurol 2019; 266: 2137-2143

[38] Pizza F, Jaussent I, Lopez R et al. Car crashes and central disorders of hypersomnolence: a French study. PLoS One 2015; 10: e0129386

[39] [Anonym]. Begutachtungsleitlinien zur Kraftfahreignung vom 27. Januar 2014 (Verkehrsblatt S. 110), Fassung vom 28.10.2019 (Verkehrsblatt S. 775), in Kraft getreten am 31.12.2019 mit der Vierzehnten Verordnung zur Änderung der Fahrerlaubnis-Verordnung und anderer straßenverkehrsrechtlicher Vorschriften. In. Bundesgesetzblatt Teil I Nr. 52 vom 30. Dezember 2019

[40] Scammell TE. Narcolepsy. N Engl J Med 2015; 373: 2654-2662

[41] Kallweit U, Bassetti CL. Pharmacological management of narcolepsy with and without cataplexy. Expert Opin Pharmacother 2017; 18: 809-817

[42] [Anonym]. Randomized trial of modafinil for the treatment of pathological somnolence in narcolepsy. US Modafinil in Narcolepsy multicenter study group. Ann Neurol 1998; 43: 88-97

[43] [Anonym]. Randomized trial of modafinil as a treatment for the excessive daytime somnolence of narcolepsy: US Modafinil in Narcolepsy multicenter study group. Neurology 2000; 54: 1166-1175

[44] Markham A. Solriamfetol: First Global Approval. Drugs 2019; 79: 785790

[45] Dauvilliers $Y$, Arnulf I, Szakacs $Z$ et al. Long-term use of pitolisant to treat patients with narcolepsy: harmony III study. Sleep 2019; 42

[46] Szakacs Z, Dauvilliers Y, Mikhaylov V et al. Safety and efficacy of pitolisant on cataplexy in patients with narcolepsy: A randomised, doubleblind, placebo-controlled trial. Lancet Neurol 2017; 16: 200-207

[47] Group XIS. A double-blind, placebo-controlled study demonstrates sodium oxybate is effective for the treatment of excessive daytime sleepiness in narcolepsy. J Clin Sleep Med 2005; 1: 391-397

[48] [Anonym]. A randomized, double blind, placebo-controlled multicenter trial comparing the effects of three doses of orally administered sodium oxybate with placebo for the treatment of narcolepsy. Sleep 2002; 25 : $42-49$

[49] Mamelak M, Black J, Montplaisir J et al. A pilot study on the effects of sodium oxybate on sleep architecture and daytime alertness in narcolepsy. Sleep 2004; 27: 1327-1334

[50] [Anonym]. A 12-month, open-label, multicenter extension trial of orally administered sodium oxybate for the treatment of narcolepsy. Sleep 2003; 26: 31-35

[51] Parkes JD, Chen SY, Clift S] et al. The clinical diagnosis of the narcoleptic syndrome. J Sleep Res 1998; 7: 41-52 